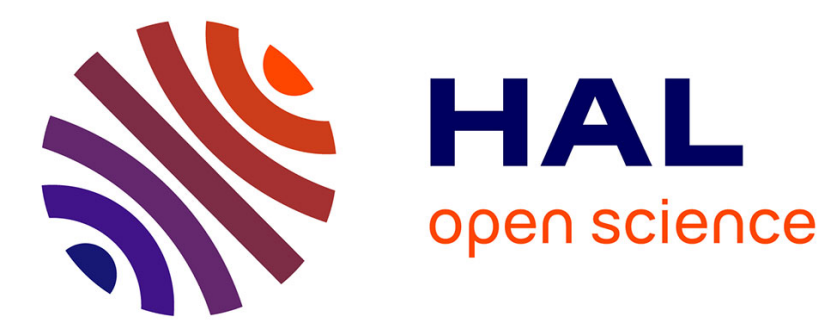

\title{
Primary lower limb lymphoedema: classification with non-contrast MR lymphography
}

Lionel Arrivé, S. Derhy, B. Dahan, S. El Mouhadi, L. Monnier-Cholley, Y. Menu, C. Becker

\section{To cite this version:}

Lionel Arrivé, S. Derhy, B. Dahan, S. El Mouhadi, L. Monnier-Cholley, et al.. Primary lower limb lymphoedema: classification with non-contrast MR lymphography. European Radiology, 2017, 10.1007/s00330-017-4948-z . hal-01578134

\section{HAL Id: hal-01578134 https://hal.sorbonne-universite.fr/hal-01578134}

Submitted on 28 Aug 2017

HAL is a multi-disciplinary open access archive for the deposit and dissemination of scientific research documents, whether they are published or not. The documents may come from teaching and research institutions in France or abroad, or from public or private research centers.
L'archive ouverte pluridisciplinaire HAL, est destinée au dépôt et à la diffusion de documents scientifiques de niveau recherche, publiés ou non, émanant des établissements d'enseignement et de recherche français ou étrangers, des laboratoires publics ou privés. 
Primary lower limb lymphedema: classification with non-contrast MR lymphography

Arrivé $\mathrm{L}^{1}$, Derhy $\mathrm{S}^{1}$, Dahan $\mathrm{B}^{2}$, El Mouhadi $\mathrm{S}^{1}$, Monnier-Cholley $\mathrm{L}^{1}$, Menu $\mathrm{Y}^{1}$, Becker $\mathrm{C}^{3}$

1 Department of Radiology, Saint-Antoine Hospital, Assistance Publique-Hôpitaux de Paris, 184 Rue du Faubourg Saint-Antoine, 75012 Paris, France / Sorbonne Universités, UPMC Université Paris 06, Faculté de Médecine Pierre et Marie Curie

2 Department of Emergency medicine, Cochin Hospital, 27 Rue du Faubourg Saint-Jacques, 75014 Paris

3 Department of Thoracic Surgery, HEGP, 20 Rue Leblanc, 75015 Paris

Corresponding author:

Lionel Arrivé

lionel.arrive@aphp.fr

Phone: 33149282258

Fax: 33149282259 


\begin{abstract}
Purpose

The purpose of the present study was to analyze the performances of non-contrast MR lymphography for the classification of primary lower limb lymphedema in 121 consecutive patients with 187 primary lower limb lymphedemas.

Materials and methods

121 consecutive patients with clinically diagnosed primary lower limb lymphedema underwent non-contrast MR lymphography with a free-breathing 3D fast spin-echo sequence with a very long TR/TE (4000/884 ms). MR examinations were retrospectively reviewed for severity of lymphedema (absent, mild, moderate, severe) and characteristics of inguinal lymph nodes and iliac and inguinal lymphatic trunks graded as aplasic (no lymph nodes or lymphatic trunks), hypoplasic (less lymph nodes or lymphatic trunks), normal and hyperplasic (more lymph nodes or more and/or dilated trunks).

Results
\end{abstract}

There was an excellent correlation between clinical stage and severity of lymphedema (Cramer's V of $0,73(\mathrm{p}<0.001))$. Differentiation was feasible between inguinal lymphatic vessel aplasia (21\%), hypoplasia (15\%), normal pattern (53\%) and hyperplasia (11\%). Severe lymphedema was observed in $46 \%$ of aplasic patterns and in $37 \%$ of hyperplasic patterns, but in only $15 \%$ of hypoplasic patterns and never observed in normal patterns $(\mathrm{p}<0.001)$

Conclusion

Non-contrast MR lymphography is able to classify primary lower limb lymphedemas into hyperplasic, aplasic, hypoplasic and normal patterns.

\title{
Keywords
}

Lower limb lymphedema 
Non-contrast MR lymphography

MR lymphography

Lymphatic vessels

Lymphedema

\section{Key points}

Non-contrast MR lymphography is able to classify primary lower limb lymphedemas

Lymphedema can be classified in hyperplasic, aplasic, hypoplasic and normal patterns

Non-contrast MR lymphography can optimize clinical management of primary lower limb lymphedemas 


\section{Introduction}

Lymphedema is a progressive condition characterized by gross swelling of the affected limb, which leads to chronic inflammation, fibrosis, and susceptibility to infection (1).

In 1934, Allen introduced a classification of lymphedema into primary and secondary. Secondary lymphedema follows obstruction to the lymphatic pathways by causes such as surgery, radiation therapy, and involvement of lymph nodes by malignant disease and other conditions (2). Although primary lymphedema is sometimes congenital, in most cases it appears early in life with a predilection for the female sex: lymphedema precox. Late onset of the disease (after age 35), known as lymphedema tarda, is uncommon (2). Primary lymphedema is an important medical issue that occurs in 1 out of every 10,000 people in the general population (3). Previous studies using conventional oil-contrast lymphography have demonstrated in primary lower limb lymphedema different patterns of lymphatic vessel abnormalities, which are designated as follows: aplasia, where there are no formed lymph trunks in the areas investigated; hypoplasia, where the lymph trunks are smaller or fewer in number than normal; and hyperplasia, where the lymph trunks of the lower limb are more numerous or greater in diameter than normal (4).

Primary lower limb lymphedema is a chronic, debilitating condition that has traditionally been seen as a refractory or incurable disorder. The conventional treatment for chronic lymphedema aims at alleviating symptoms, and is mainly based on physiotherapy and/or controlled compression therapy. However, with advances in our understanding of lymphedema pathophysiology, as well as new diagnostic and therapeutic tools, this perspective has changed. New surgical approaches include free lymph node autologous transplantation for lymphatic reconstruction and multiple lymphaticovenular anastomoses to derive the excess fluid component (5-7). In essence, lymph node transplantation could be more appropriate for aplasic or hypoplasic primary lymphedema, and lymphaticovenular 
anastomoses for hyperplasic primary lymphedema (5-7). In addition, in animal models, it has been demonstrated that vascular endothelial growth factor C (VEGF-C) or VEGF-D may induce growth of the lymphatic capillaries $(8,9)$.

Patients with primary lymphedema should undergo proper imaging to evaluate the variety and characteristics of lymphedema and to allow for optimal clinical management and appropriate selection of treatment. However, the ideal imaging technique for the evaluation of patients with primary lymphedema has been elusive. The invasiveness and the morbidity of conventional oil-contrast lymphangiography have resulted in a dramatic decrease of examinations (10). The current primary imaging modality for the investigation of peripheral lymphedema is lymphoscintigraphy performed with 99mTc-labeled nanocolloids. Lymphoscintigraphy provides insights into lymph flow dynamics. However, it is also hampered by low spatial resolution (11). The principle of contrast-enhanced MR lymphangiography is to subcutaneously inject paramagnetic macromolecules that enter the lymphatic vessels, resulting in contrast enhancement on T1-weighted MR sequences $(12,13)$. The spatial resolution is far higher than that of lymphoscintigraphy (14). Enhancement of lymphatic vessels is optimal near the contrast media site. On the other hand, visualization of larger lymphatic vessels may be suboptimal, and venous enhancement which is frequently observed may be confusing $(15,16)$.

Non-contrast heavily T2-weighted 3D MRI, which was introduced in the early 1990s, is a non-invasive imaging technique that can accurately depict the morphological features of the biliary and pancreatic ducts. By using heavily T2-weighted sequences without intravenous administration of any contrast agent, the signal of static or slow-moving fluid-filled structures, such as the lymphatic vessels, results in unique lymphatic vessels to background contrast. Though MR imaging with heavily T2-weighted MR images has already been used for the 
morphological diagnosis of lymphedema, analysis of lymphatic vessels has not yet been performed (10, 17-19).

The purpose of the present study was to analyze the performance of non-contrast MR lymphography for the classification of primary lower limb lymphedema in 121 consecutive patients clinically suspected to have lymphedema.

Materials and methods

\section{A. Patients}

From March 2011 to March 2015, 121 consecutive patients with clinically diagnosed primary lower limb lymphedema were referred to our institution for non-contrast MR lymphography. Our local institutional review board approved this study. Informed consent from patients was not required for this observational retrospective study. In 14 patients, no lymphedema was demonstrated with non-contrast MR lymphography. In these 14 patients MR lymphography only demonstrated excessive subcutaneous deposition of fat related to lipedema or morbid obesity without any lymphedema. These 14 patients were therefore excluded from further analysis. The remaining 107 patients (90 female and 17 male patients) had a mean age of 37.6 +/- 15.2 years. The mean duration of limb swelling was $20.1+/-17.4$ years. There were 84 lymphedema precox (onset before age 35) and 23 lymphedema tarda (onset after age 35) patients. Clinical diagnosis and clinical staging were established by a lymphologist surgeon (CB) with more than 20 years of experience.

Clinical staging was based upon the criteria established by the International Society of Lymphology in 2009 (20) (Table 1). All possible secondary causes of lymphatic disease had been excluded by a complete work up before considering the diagnosis of primary lymphedema. Furthermore, in all cases primary lower limb lymphedema was solitary and not 
as part of a generalized lymphatic anomaly. All patients had conservative management including physiotherapy and compression therapy but no patient had previous surgery.

\section{B. MR examination}

All MR examinations were performed with a 1.5 Tesla General Electric Signa HDxt (GE Medical System, Wisconsin, USA). Five hundred $\mathrm{ml}$ of pineapple juice, used as a negative oral contrast agent to decrease bowel content signal intensity, was given 30 minutes before MR examination. The patients were placed in a supine position with their feet first. Three successive acquisitions were performed on the lower leg and foot (first station), thigh (second station), and the pelvic areas (third station), with six-element phased-array coils.

a) A T1-weighted three-plane localizer was performed for orientation purposes for each station.

b) Two stacks of T2-weighted MR images using IDEAL (Iterative Decomposition of water and fat with Echo Asymmetry and Least-squares estimation) (fat/water separation technique providing four different contrasts: water, fat, in phase and out phase) T2 FSE (TR/TE; 4233/76 ms) sequences (slice thickness: 6 mm; matrix: 320x192; FOV: 380x380; scan time 3 minutes 30 seconds) were performed in the axial plane. The first stack of images was obtained on the pelvic area for inguinal nodes analysis and the second stack of images was obtained on the legs for lymphedema characteristics analysis.

c) Non-contrast MR lymphography was obtained with a free-breathing 3D high spatial resolution fast spin-echo sequence similar to that used for 3D MR cholangiopancreatography, with a very long TR/TE (4000/884 ms; flip angle $\left.90^{\circ}\right)$ in the coronal plane. A $90^{\circ}$ radiofrequency pulse was applied at the end of the echo train to flip the transverse magnetization to the longitudinal direction. The matrix was $512 \times 288$ with a field of view of $400 \mathrm{x} 400$. The section thickness varied from 0.8 to $1.4 \mathrm{~mm}$. A stack of 
124 to 316 sections was acquired to cover the entire region of interest. Scan time varied from 3 to 5 minutes depending of the number of native slices. Post-processing of the data was performed to obtain maximum intensity projection (MIP) images and multiplanar reformatted images.

C. Image analysis

All MR examinations were retrospectively reviewed in conference by two radiologists (LA with 10 years of experience and SD with seven years of experience in non-contrast MR lymphography). Agreement was reached by consensus. MR images were analyzed on a PACS system (Carestream version 11.32, Carestream Health, Rochester, NY). For 3D MR lymphography thin source images, 10-mm MIP and volume MIP reconstructed images were evaluated. Observers were blinded to clinical lymphedema staging.

Criteria of analysis included:

a) Severity of lymphedema graded as absent, mild, moderate and severe.

Lymphedema was graded as mild when: a) superior margin of subcutaneous infiltration was below the knee; and b) fluid infiltration of subcutaneous fat was mild without increase of the dimensions of subcutaneous fat; and c) epifascial fluid collection was minimal (less than $5 \mathrm{~mm}$ of thickness).

Lymphedema was graded as severe when: a) subcutaneous infiltration involved the entire lower limb; and b) fluid infiltration was severe with marked increase of the dimension of subcutaneous fat; and c) epifascial fluid collection was severe (more than $15 \mathrm{~mm}$ of thickness).

Lymphedema was graded as moderate for all intermediate situations between mild and severe. 
b) Presence or absence of the "so-called" honeycomb pattern corresponding to trabecular structure with enlarged fat pockets surrounded by thick lines (21).

c) Presence or absence of increased thickness of dermis (dermis thickness greater than 2 $\mathrm{mm})$.

d) Presence or absence of involvement of muscular compartment (fluid infiltration, fatty muscular involution, or atrophy).

e) Number of inguinal lymph nodes graded as: no nodes; decreased number: fewer than 3 nodes; normal: 3 to 6 nodes; increased number: 7 or more nodes.

f) and g) Characteristics of iliac (f) and inguinal (g) lymphatic trunks graded as aplasic when no lymphatic trunks were demonstrated; hypoplasic when fewer than 3 lymphatic trunks were demonstrated; normal when 3 to 6 lymphatic trunks were demonstrated; and hyperplasic when more than 6 lymphatic trunks were demonstrated, or when lymphatic trunks were abnormally increased in diameter (lymphatic trunk diameter $\geq 3 \mathrm{~mm}$ ).

h) Presence or absence of distal dilated lymphatic vessels (lymphatic vessel diameter superior to $1 \mathrm{~mm}$ ) in the leg.

D. Statistical analysis

We reported continuous variables as means (+/- SD) and categorical variables as proportions. We used Student's t-test to analyze between-group differences as appropriate. A chi-square test was used for categorical variables with expected values more than 5, and Fisher's exact test was used for categorical variables with expected values less than 5. We used Cramer's V coefficient to measure the correlation between two nominal variables. All analyses were performed with the use of SAS software, version 9.3 (SAS Institute). A two-sided p value of 0.05 or less was considered to indicate statistical significance. 
Results

All 121 patients completed the MR examination successfully. For the 107 patients with lymphedema demonstrated with MR imaging, inguinal lymph nodes and inguinal lymphatic vessels could not accurately be analyzed in four patients, and iliac lymphatic vessels in another six patients, due to suboptimal positioning of the phase-array coil in the third station of the examination.

Lymphedema was bilateral in 80 patients and unilateral in the other 27 , resulting in 187 lower limb lymphedemas (Table 2). Clinical stages were I in 85 lower limbs (45\%), II in 78 (42\%), and III in the other 24 (13\%) (Figure 1a) (Table 3). Familial history of lymphedema was reported in 13 patients. History of erysipelas was reported in 12 patients (18 lower limbs) With non-contrast MR lymphography, lymphedema was mild in 110 (59\%) lower limbs, moderate in $44(23 \%)$, and severe in the other 33 (18\%) (Figures 1-5) (Table 3). There was an excellent correlation between the clinical stage and the severity of lymphedema, with a Cramer's V of $0.73(\mathrm{p}<0.001)($ Table 3$)$

A so-called honeycomb pattern was present in 120 lower limbs and absent in the other 67 (Figure 2d). It was more common in severe lymphedema $(\mathrm{p}<0.001)$. Increased thickness of dermis was present in only 35 lower limbs (19\%) (Figure 2d). Involvement of the muscular compartment was never observed. Inguinal lymph nodes were absent in $11(6 \%)$ lower limbs, decreased in $19(11 \%)$, normal in $144(80 \%)$, and increased in six (3\%) (Figure 4c). Iliac and inguinal lymph trunks, respectively, were absent (aplasic pattern) in $36(22 \%)$ and $37(21 \%)$ (Figure 4a), decreased (hypoplasic pattern) in 25 (15\%) and 27 (15\%) (Figure 1b), normal in $94(56 \%)$ and $96(53 \%)$ (Figure 5a), and increased in size or number (hyperplasic or dysplasic pattern) in $11(7 \%)$ and $19(11 \%)$ (Figures 2a, 3a). There was a positive correlation between number of inguinal lymph nodes and number of both iliac and inguinal lymphatic trunks $(p<0.001)$. The severity of lymphedema was positively correlated with the characteristics of 
inguinal lymph nodes, iliac and inguinal lymphatic trunks (i.e; aplasia, hypoplasia, normal and hyperplasia) as demonstrated with non-contrast MR lymphography $(\mathrm{p}<0.001)$. Severe lymphedema was observed in $46 \%$ of aplasic patterns and in $37 \%$ of hyperplasic patterns, but in only $15 \%$ of hypoplasic patterns and never observed in a normal pattern $(p<0.001)$ (Table 4).

Distal dilated lymphatic vessels in the leg were observed in 68 (36\%) cases. Dilatation of distal lymphatic vessels in the leg was observed in all cases of hyperplasic patterns, but also in $29 \%$ of the other patterns (Figures 2b, 2c, 3b, 3c). In contrast, we did not find a significant correlation between distal lymphatic vessel dilatation and duration of limb swelling. Similarly, we did not find any significant association between patient age, sex, duration of swelling, history of erysipelas or familial history lymphedema and characteristics of lower limb lymphedema as demonstrated with non-contrast MR lymphography.

\section{Discussion}

Lymphedema is defined as accumulation of protein-rich lymph in the interstitial space caused by failure of the lymphatic system to conduct lymph back to the blood circulation. This failure to transport excess interstitial fluid and large molecular substances such as proteins, fat and waste material from the interstitium results in development of fibrosis in the dermis in chronic lymphedema $(1,10,20)$. Fibrotic changes with inflammatory cells in the dermis and proliferation of collagen fibers occurring in the subcutaneous compartment which is the site of most of the swelling contribute to the solid tissue consistency $(1,20)$. With non-contrast MR lymphography, primary lower limb lymphedema is demonstrated as a combination of fluid infiltration of subcutaneous fat and epifascial fluid collection. The fluid infiltration of 
subcutaneous fat commonly exibits the "so-called" honeycomb pattern which corresponds to trabecular structure with enlarged fat pockets surrounded by lines corresponding to fluid or fibrous tissue (fibrous strands due to chronic inflammation are caused by the high protein content of edema fluid) or both (21). These features allow for an obvious differentiation from fatty infiltration related to lipedema or morbid obesity $(1,3)$.

It is also easy to differentiate lymphedema from phlebedema; in the latter condition, there is an involvement of the subfascial muscular compartment with increase in size with muscle edema or fatty muscular degeneration $(21,22)$. Such involvement of the subfascial muscular compartment was never observed in our series of 187 lower limb lymphedemas, confirming that lymphedema is primarily a disorder of subcutaneous tissue. In most cases, diagnosis of lymphedema can be made on the basis of medical history and physical examination. However, it is not always easy to clinically distinguish between lymphedema and other types of edema, such as phlebedema or lipedema $(23,24)$. In our study, lymphedema was not demonstrated with non-contrast MR lymphography in 14 patients clinically diagnosed with lymphedema. Only fatty infiltration related to lipedema or morbid obesity was demonstrated in these 14 patients. However, in the other 107 patients, there was an excellent correlation between the clinical stage and the severity of lymphedema, as demonstrated with non-contrast MR lymphography. The level of detail of soft tissue contrast obtained with non-contrast MR lymphography allows for an excellent delineation of soft tissue edema localization, which may help to optimize mapping of the manual lymphatic drainage.

Iliac and inguinal lymphatic trunks were aplasic in $22 \%$ and $21 \%$ of cases, hypoplasic in $15 \%$ of cases, and hyperplasic in $7 \%$ and $11 \%$ of cases. They were considered normal in $56 \%$ and $53 \%$ of cases. We also found a positive correlation between lymphatic trunk characteristics and inguinal lymph node number. 
In 1957, Kinmonth et al. found aplasia in $14 \%$ of cases, hypoplasia in $55 \%$ of cases, and hyperplasia in $24 \%$ of cases in a study of 87 patients with primary lymphedema (4). By means of X-ray lymphangiography, lymphatic vessels were only considered normal in $6 \%$ of cases. Hypoplasia was more common in their study than in ours. However, lymphatic vessels of the entire upper and lower leg were analyzed by X-ray lymphangiography, whereas in our study, only lymphatic trunks were analyzed. Lymphatic vessels distal to the lymphatic trunks were not analyzed because of limited spatial resolution of non-contrast MR lymphography. In addition, in their 1957 study, Kinmonth et al. classified cases with lymph trunk deficient either in number or in size as hypoplasic (4). Because of limited spatial resolution of noncontrast MR lymphography in comparison with X-ray lymphangiography, we only evaluated the number of lymph trunks or the increase in size. Because X-ray lymphangiography is no longer performed, and because to the best of our knowledge no other imaging technique allows for a reliable analysis of lymphatic trunks, we do not have any means for comparing our results to more recent data. One can imagine that recruitment of patients in 1957 was markedly different from our own recruitment.

We found an excellent positive correlation between the severity of lymphedema and characteristics of lymph trunks. Lymphedema was more severe in case of aplasic and hyperplasic patterns. It was less severe in cases of lymphedema with hypoplasic or normal iliac and inguinal lymphatic trunks. According to us such correlation not previously demonstrated reinforces the interest of the classification allowed by non-contrast MR lymphography. Interestingly, we did not find any correlation between dilatation of distal lymphatic vessels and duration of limb swelling. It is frequently considered that in longstanding lymphedema lymphatic dilatation is no more observed because of marked thickening of lymphatic wall; our study did not confirm this hypothesis (25). 
In the past, lymphedema was frequently considered to be a refractory or incurable condition, and treatment was aimed to decrease symptoms with physiotherapy and/or compression therapy. However, new surgical approaches are now available. We assume that a detailed anatomic evaluation of both lymph nodes and lymphatic vessels may be useful for surgical planning. The choice of site for free lymph node autologous transplantation may be optimized by the precise evaluation of the extent of lymphedema and by the characteristics of abnormalities of lymph nodes and lymphatic vessels. Lymph node transplantation appears to be an interesting surgical modality in cases of hypoplasic or aplasic lymphedema $(6,26)$. On the other hand, lymphaticovenular anastomosis appears to be an interesting surgical modality in cases of dilated lymphatic vessels without hypoplasia. Presurgical evaluation needs a precise delineation of the lymphatic drainage system in the targeted limb, including number, caliber, and location. In contrast, the outcome of lymphaticovenular anastomosis may be weaker for patients with atrophied or sclerotic lymphatic vessels (27).

The optimal imaging modality for evaluation of lower limb lymphedema has not yet been established. Because of its invasiveness compared with new techniques, conventional oilcontrast X-ray lymphangiography is no longer performed in patients with lower limb lymphedema $(10,28)$. The current primary imaging modality is lymphoscintigraphy. Lymphoscintigraphy can detect retarded tracer transport even in mild lymphedema and is useful to evaluate the functional lymph flow. Quantitative analysis of lymph transport is performed by obtaining dynamic images or measuring the transit time $(10,11)$. On the other hand suboptimal resolution of lymphoscintigraphy is not suitable for demonstrating normal size lymphatic vessels $(10,11)$.Contrast-enhanced MR lymphangiography has a far higher spatial resolution (29), and its evaluation of distal lymphatic vessels near the site of contrast injection is excellent. Both contrast enhanced MR lymphangiography and lymphoscintigraphy provide dynamic information of contrast agent uptake. Notohamiprodjo et al. demonstrated 
that the two techniques were strongly correlated for depiction of delayed and diffuse lymphatic drainage in peripheral lymphedema (29). However, the visualization of the larger lymphatic vessels, including the inguinal and iliac lymphatic trunks, is of lower quality than the distal lymphatic trunks, and the enhancement of the pelvic lymphatic vessels is usually poor (30). In addition, a substantial amount of contrast material is eliminated via the circulating system, and enhancement of the venous system may complicate analysis of the lymphatic vessels. To improve differentiation from venous structures, Mitsumori et al. (14) added contrast enhanced MR venography. In addition enhanced lymphatic vessels may not be demonstrated in healthy limbs, probably because of a faster lymphatic flow (14). Finally, in comparing contrast-enhanced MR lymphangiography and heavily T2-weighted MRI, Lu et al. demonstrated that number and diameter of lymphatic vessels were different (31). They postulated that uptake of contrast media may be restricted in dilated lymphatic vessels because of increased lymphatic pressure. On the other hand, contrast-enhanced MR lymphangiography allows a functional assessment of the lymphatic vessels transportation and nodal uptake. In a study published in 2005, Liu et al. reported the results of non-contrast 3D MR imaging for the evaluation of lymph circulation disorders. However, because of the limited spatial resolution, they reported that such imaging was not feasible for the depiction of lymphatic vessels in an intact lymphatic system (32). On the other hand, several studies have demonstrated that larger lymphatic trunks, such as retroperitoneal lymphatic trunks, were easily demonstrated with non-contrast MR lymphography (33-35). Yamamoto and colleagues reported the results of Indocyanine Green (ICG) lymphography in 31 cases of primary lower limb lymphedema (36). They were able to differentiate four different patterns including proximal and distal backflow, less and no enhancement patterns. ICG lymphography allowed for the identification of superficial lymphatic vessels. Therefore, the main advantage of ICG lymphography is the potential for real-time observation of lymphatic vessels during surgery. 
Limitations of ICG lymphography include relatively small field of view and markedly limited penetration depth, therefore ICG lymphography cannot be used to analyze deep lymphatic vessels (36). In contrast with these techniques non contrast MR lymphography does not provide any functional information, however the precise anatomic information can be used to identify sites for lymph node transplantation or suitable sites for lympho-venous anastomosis and to optimize mapping of the manual lymphatic drainage. We believe that it could be very interesting to associate non-contrast MR lymphography with a functional imaging modality such as contrast-enhanced MR lymphangiography as already proposed by Mitsumori et al (14). In addition, contrast- enhanced MR lymphangiography appears to be optimal for evaluation of distal lymphatic vessels whereas non-contrast MR lymphography may be superior for evaluation of proximal lymphatic trunks including inguinal and iliac vessels. The main limitation of the present study is the lack of a comparative imaging modality and the definite lack of a standard of reference. As a matter of fact, in our retrospective study comparative imaging modality was uncommonly available. However, the results obtained with non-contrast MR lymphography are fundamentally different from those obtained by other imaging modalities except $\mathrm{X}$ ray lymphangiography which is no more performed in patients with primary lymphedema. In addition, since it is a single-center retrospective study, we cannot be certain of the representability of the population evaluated. Several image analysis criteria were only based upon our experience of non-contrast MR lymphography and the reading was only performed in consensus. For example, we considered it to be normal to see between three and six lymphatic trunks in the inguinal area, when it is usually normal to find between five and 15 lymphatic vessels with X-ray lymphangiography, due to its greater spatial resolution (4). Our preliminary results should be externally validated by other centers' experiences. Finally, as non-contrast MR lymphography is a relatively new imaging 
technique, its main limitation today is its suboptimal spatial resolution, which may be improved upon in the near future with advances in the software and hardware of MR systems.

In summary we believe that non-contrast MR lymphography, which is a perfectly noninvasive imaging technique, is able to differentiate primary lower limb lymphedema in hyperplasic, aplasic, hypoplasic patterns and can potentially optimize clinical management. 
Acknowledgment: Julien Bouvier for technical assistance. 


\section{References}

1. Cho S, Atwood JR. Peripheral edema. Am J Med 2002;113:580-586

2. Allen EV. Lymphedema of the extremities. Classification, etiology and differential diagnosis: a study of three hundred cases. Arch Int Med 1934;54:606-624

3. Petrek JA, Pressman PI, Smith RA. Lymphedema: current issues in research and management. CA Cancer J Clin 2000;50:292-307

4. Kinmonth JB. Primary lymphoedema. Clinical and lymphangiographic studies of a series of 107 patients in which the lower limbs were affected. Br J Surg 1957;189:1-9

5. Campisi C, Davini D, Bellini C et al. Lymphatic microsurgery for the treatment of lymphedema. Microsurgery 2006;26:65-69

6. Becker C, Assouad J, Riquet M, Hidden G. Postmastectomy lymphedema: long-term results following microsurgical lymph node transplantation. Ann Surg 2006;243:313-315

7. Olszewski WL. Lymphovenous microsurgical shunts in treatment of lymphedema of lower limbs: a 45-year experience of One surgeon/One center. Eur J Vasc and Endovasc Surg 2013;45:282-290

8. Tammela T, Saaristo A, Holopainen T et al. Therapeutic differentiation and maturation of lymphatic vessels after lymph node dissection and transplantation. Nature Medicine 2007;13:1458-1466

9. Lähteenvuo M, Honkonen K, Tervala T et al. Growth factor therapy and autologous lymph node transfer in lymphedema. Circulation 2011;123:613-620

10. Witte CL, Witte MH, Unger EC. Advances in imaging of lymph flow disorders. RadioGraphics 2000;20:1697-1719 
11. Weissleder H, Weissleder R. Lymphedema: evaluation of qualitative and quantitative lymphoscintigraphy in 238 patients. Radiology 1988;167:729-735

12. Ruehm SG, Schroeder T, Debatin JF. Interstitial MR lymphography with gadoterate meglumine: initial experience in humans. Radiology 2001;220:816-821

13. Lohrmann C, Foeldi E, Speck O, Langer M. High-resolution MR lymphangiography in patients with primary and secondary lymphedema. AJR Am J Roentgenol 2006;187:556561

14. Mitsumori LM, McDonald ES, Wilson GJ, Neligan PC, Minoshima S, Maki HM. MR lymphangiography: How I do it. J Magn Reson Imag 2015;42:1465-1477

15. Lu Q, Delproposto Z, Hu A et al. MR lymphography of lymphatic vessels in lower extremity with gynecologic oncology-related lymphedema. PLOS One 2012;7:e50319

16. Liu NF, Lu Q, Jiang ZH, Wang CG. Anatomic and functional evaluation of the lymphatic and lymph nodes in diagnosis of lymphatic circulation disorders with contrast magnetic resonance lymphangiography. J Vasc Surg 2009;49: 980-987

17. Case TC, Witte CL, Witte MH, Unger EC, Williams WH. Magnetic resonance imaging in human lymphedema: comparison with lymphangioscintigraphy. Magn Reson Imaging $1992 ; 10: 549-558$

18. Liu NF, Wang CG. The role of magnetic resonance imaging in diagnosis of peripheral lymphatic disorders. Lymphology 1998;31:119-127

19. Aström KGO, Abdsaleh S, Brenning GC, Ahlström KH. MR imaging of primary, secondary, and mixed forms of lymphedema. Acta Radiol 2001;42:409-416

20. 2009 Consensus Document of the International Society of Lymphology. The diagnosis and treatment of peripheral lymphedema. Lymphology 2009;42:51-60 
21. Hadjis NS, Carr DH, Banks L, Pflug JJ. The role of CT in the diagnosis of primary lymphedema of the lower limb. AJR 1985;144:361-364

22. Witte CL, Witte MH. Physiology and imaging of the peripheral lymphatic system. In: Moore WS, ed. Vascular surgery. 5th ed. Philadelphia. Pa: Saunders, 1998;808-828

23. Witte MH, Witte CL. Massive obesity simulating lymphedema. N Engl J Med $1992 ; 327: 1927$

24. Duewell S, Hagspiel KD, Zuber J, von Schulthess GK, Bollinger A, Fuchs WA. Swollen lower extremity: role of MR imaging. Radiology 1992;184:227-231

25. Mihara M, Hara H, Hayashi Y et al. Pathological steps of cancer related lymphedema histological changes in the collecting lymphatic vessels after lymphadenectomy. PLOS ONE 2012;7: e41126

26. Vaqueiro M, Gloviczki P, Fisher J, Hollier LH, Schirger A, Wahner HW. Lymphoscintigraphy in lymphedema: an aid to microsurgery. J Nucl Med 1986;27:11251130

27. Maegawa J, Mikami T, Yamamoto Y, Satake T, Kobayashi S. Types of lymphoscintigraphy and indications for lymphaticovenous anastomosis. Microsurgery 2010;30:437-442

28. Koehler PR. Complications of lymphography. Lymphology 1968;1:116-120

29. Notohamiprodjo M, Weiss M, Baumeister RG et al. MR lymphangiogrpahy at 3.0T : correlation with lymphoscintigraphy. Radiology 2012;264:78-87

30. Olszewski WL, Liu NF. Magnetic resonance lymphography (MRL). Point and counter point. Lymphology 2013;46:202-207 
31. Lu Q, Xu J, Liu N. Chronic lower extremity lymphedema: A comparative study of highresolution interstitial MR lymphangiography and heavily T2-weighted MRI. Eur J Radiol 2010; 73: 365-373

32. Liu N, Wang C, Sun M. Noncontrast three-dimensional magnetic resonance imaging vs lymphoscintigraphy in the evaluation of lymph circulation disorders: a comparative study. J Vasc Surg 2005;41:69-75

33. Takahashi H, Kuboyama S, Abe H, Aoki T, Miyazaki M, Nakata H. Clinical feasibility of non-contrast enhanced magnetic resonance lymphography of the thoracic duct. Chest $2003 ; 124: 2136-2142$

34. Arrivé L, Azizi L, Lewin M et al. MR lymphography of abdominal and retroperitoneal lymphatic vessels. AJR Am J Roentgenol 2007;189:1051-1058

35. Derhy S, El Mouhadi S, Ruiz A, Azizi L, Menu Y, Arrivé L. Non-contrast 3D MR lymphography of retroperitoneal lymphatic aneurysmal dilatation: a continuous spectrum of change from normal variants to cystic lymphangioma. Insights Imaging 2013;4:753758

36. Yamamoto T, Yoshimatsu H, Narushima M, Yamamoto N, Hayashi A, Koshima I. Indocyanine Green Lymphography findings in primary leg lymphedema, Eur J Vasc Endovasc Surg 2015; 49: 95-102 


\section{Table 1: Clinical staging of lymphedema}

Stage $0 \quad$ Latent or sub-clinical condition

Swelling is not present

Stage I Early accumulation of fluid which subsides with limb elevation

Pitting may occur

Stage II Limb elevation alone rarely reduces tissue swelling

Pitting is manifest

Stage III Pitting is absent

Trophic skin changes are apparent 
Table 2: Flow chart of patients with primary lower limb lymphedema

121 patients with lymphedema at clinical examination
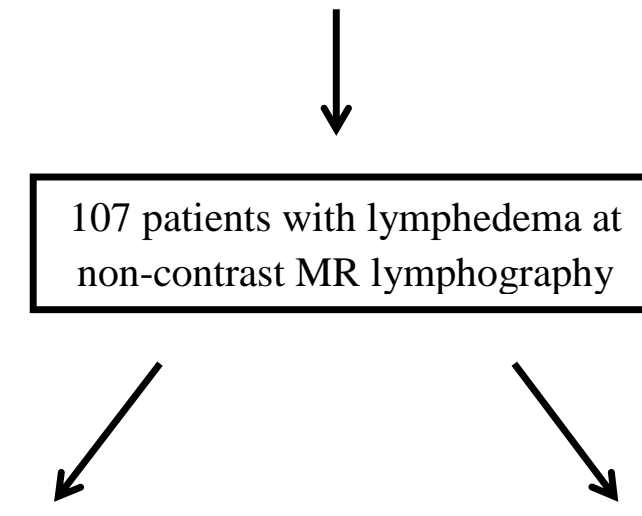

80 patients with bilateral lymphedema

27 patients with unilateral lymphedema
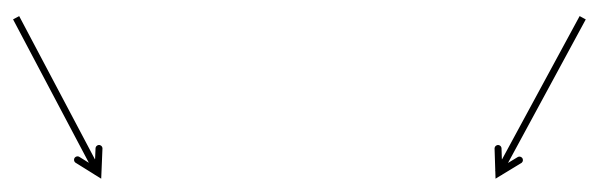

187 lower limb lymphedemas 
Table 3: Correlation between stage and severity of lymphedema demonstrated by MRI (Cramer's V of 0.73 , p<0.001)

\begin{tabular}{|c|c|c|c|c|c|}
\hline & & \multicolumn{3}{|c|}{ Severity of lymphedema with MRI } & \\
\hline & & Mild & Moderate & Severe & \\
\hline \multirow{3}{*}{ 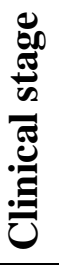 } & 1 & 83 & 0 & 2 & 85 \\
\hline & 2 & 27 & 43 & 8 & 78 \\
\hline & 3 & 0 & 1 & 23 & 24 \\
\hline & & 110 & 44 & 33 & \\
\hline
\end{tabular}


Table 4: Correlation between severity of lymphedema and pattern of inguinal lymphatic vessels (Fisher test: $\mathbf{p}<0.001$ ) (Missing data: 8). Severe lymphedema was more observed in aplasic $(46 \%)$ and hyperplasic pattern $(37 \%)$ than in hypoplasic $15 \%)$ and normal pattern $(0 \%)$.

\begin{tabular}{|c|c|c|c|c|c|c|}
\hline & \multicolumn{4}{|c|}{ Pattern of inguinal lymphatic vessels } & \\
\hline & & Aplasic & Hypoplasic & Normal & Hyperplasic & \\
\hline \multirow{3}{*}{ 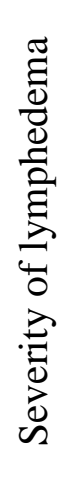 } & Mild & $\begin{array}{r}8 \\
22 \% \\
\end{array}$ & $\begin{array}{r}14 \\
52 \% \\
\end{array}$ & $\begin{array}{c}83 \\
86 \% \\
\end{array}$ & $\begin{array}{c}2 \\
10 \% \\
\end{array}$ & 107 \\
\hline & Moderate & $\begin{array}{r}12 \\
32 \% \\
\end{array}$ & $\begin{array}{r}9 \\
33 \% \\
\end{array}$ & $\begin{array}{r}13 \\
14 \% \\
\end{array}$ & $\begin{array}{r}10 \\
53 \% \\
\end{array}$ & 44 \\
\hline & Severe & $\begin{array}{r}17 \\
46 \% \\
\end{array}$ & $\begin{array}{r}4 \\
15 \% \\
\end{array}$ & $\begin{array}{c}0 \\
0 \% \\
\end{array}$ & $\begin{array}{r}7 \\
37 \% \\
\end{array}$ & 28 \\
\hline & & 37 & 27 & 96 & 19 & 179 \\
\hline
\end{tabular}


Figures legends

Figure 1: 28-year-old female with lower limb lymphedema. Clinical picture of lower limb demonstrates a severe (stage III) left lower limb lymphedema (a). Non-contrast MR lymphography demonstrates only one iliac and inguinal lymphatic trunk on the left side (arrows) and a normal pattern (arrowhead) on the right side (b). A moderate fluid infiltration (I) of subcutaneous fat is demonstrated on the left side. At lower levels (c, d), a severe fluid infiltration (I) of subcutaneous fat is demonstrated on the left side. A mild subcutaneous infiltration (arrow) not clinically detected is demonstrated on the right side. Water IDEAL T2 FSE image (e) demonstrates a severe fluid infiltration (I) of subcutaneous fat and an epifascial fluid collection (C) on the left side.

Figure 2: 54-year-old man with moderate hyperplasic bilateral lower limb lymphedema. Noncontrast MR lymphography demonstrates an increased number of dilated lymphatic iliac and inguinal trunks (arrows) (a). (Note the presence of bilateral hydrocele $(\mathrm{H})$ testis). Dilated lymphatic vessels (arrows) are demonstrated at lower levels (b, c). Fluid infiltration (I) of subcutaneous fat is also well demonstrated (b, c). Water IDEAL T2 FSE image (d) demonstrates a bilateral fluid infiltration (I) of subcutaneous fat with a so-called honeycomb pattern, moderate epifascial fluid collection (C), and an increased thickness of dermis (arrow).

Figure 3: 26-year-old man with left mild hyperplasic lower limb lymphedema. Non-contrast MR lymphography demonstrates an increased number of dilated left lymphatic iliac and inguinal trunks (arrows) (a). Dilated lymphatic vessels are demonstrated at lower levels (arrows) (b, c). Mild fluid infiltration of subcutaneous fat is demonstrated within the leg maximal above the medial side of ankle (arrowhead) (c). Marked dilatation of lymphatic 
vessels is demonstrated around the popliteal lymph node (short arrow) (b). Neither lymphatic abnormality nor lymphedema were observed on the right side.

Figure 4: 18-year-old man with left moderate aplasic lower limb lymphedema. Non-contrast MR lymphography demonstrates the absence of iliac and inguinal lymphatic trunks. Normal appearance of right iliac and inguinal lymphatic trunks (arrows). Mild fluid infiltration (I) of medial part of left thigh is also demonstrated (a). Moderate fluid infiltration (I) of left leg is also demonstrated (b). Small superficial lymphatic vessels (arrow) are demonstrated on the medial side of left knee (b). Water IDEAL T2 FSE image demonstrates absence of left inguinal lymph node (c) whereas right inguinal lymph nodes (arrows) are normal and a moderate fluid infiltration (I) of lower limb without epifascial fluid collection of left lower $\operatorname{limb}(d)$.

Figure 5: 52-year-old female with mild lower limb lymphedema. Non-contrast MR lymphography demonstrates a normal pattern of iliac and inguinal lymphatic trunks (arrows) at both sides (a). A lower levels (b, c), a bilateral mild fluid infiltration (I) of subcutaneous fat is demonstrated. 